PREPARED FOR THE U.S. DEPARTMENT OF ENERGY, UNDER CONTRACT DE-AC02-76CH03073

PPPL-3721

PPPL-3721

UC-70

Microwave Imaging Reflectometer for TEXTOR

by

T. Munsat, E. Mazzucato, H. Park, B.H. Deng, C.W. Domier, N.C. Luhmann, Jr., J. Wang, Z.G. Xia, A.J.H. Donné, and M. van de Pol

July 2002

NM|

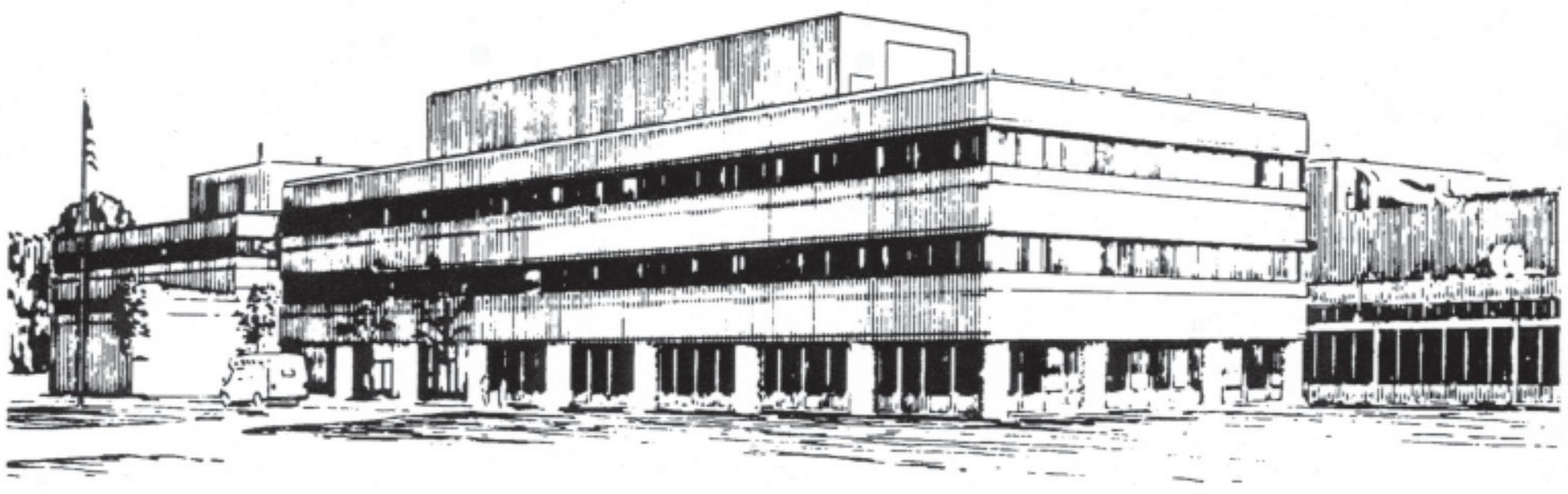

PRINCETON PLASMA PHYSICS LABORATORY PRINCETON UNIVERSITY, PRINCETON, NEW JERSEY 


\section{PPPL Reports Disclaimer}

This report was prepared as an account of work sponsored by an agency of the United States Government. Neither the United States Government nor any agency thereof, nor any of their employees, makes any warranty, express or implied, or assumes any legal liability or responsibility for the accuracy, completeness, or usefulness of any information, apparatus, product, or process disclosed, or represents that its use would not infringe privately owned rights. Reference herein to any specific commercial product, process, or service by trade name, trademark, manufacturer, or otherwise, does not necessarily constitute or imply its endorsement, recommendation, or favoring by the United States Government or any agency thereof. The views and opinions of authors expressed herein do not necessarily state or reflect those of the United States Government or any agency thereof.

\section{Availability}

This report is posted on the U.S. Department of Energy's Princeton Plasma Physics Laboratory Publications and Reports web site in Fiscal Year 2002. The home page for PPPL Reports and Publications is: http://www.pppl.gov/pub_report/

DOE and DOE Contractors can obtain copies of this report from:

U.S. Department of Energy

Office of Scientific and Technical Information

DOE Technical Information Services (DTIS)

P.O. Box 62

Oak Ridge, TN 37831

Telephone: (865) 576-8401

Fax: (865) 576-5728

Email: reports@adonis.osti.gov

This report is available to the general public from:

National Technical Information Service

U.S. Department of Commerce

5285 Port Royal Road

Springfield, VA 22161

Telephone: 1-800-553-6847 or

(703) 605-6000

Fax: (703) 321-8547

Internet: http://www.ntis.gov/ordering.htm 


\title{
Microwave Imaging Reflectometer for TEXTOR
}

\author{
T. Munsat, E. Mazzucato, and H. Park \\ Plasma Physics Laboratory, Princeton University, Princeton, NJ 08543 \\ B.H. Deng, C.W. Domier, N.C. Luhmann, Jr., J. Wang, and Z.G. Xia \\ University of California, Davis
}

A.J.H. Donné and M. van de Pol

FOM Institute for Plasma Physics "Rijnhuizen", The Netherlands

(Dated: July 5, 2002)

\begin{abstract}
Understanding the behavior of fluctuations in magnetically confined plasmas is essential to the advancement of turbulence-based transport physics. Though microwave reflectometry has proven to be an extremely useful and sensitive tool for measuring small density fluctuations in some circumstances, this technique has been shown to have limited viability for large amplitude, high $k_{\theta}$ fluctuations and/or core measurements. To this end, a new instrument based on 2-D imaging reflectometry has been developed to measure density fluctuations over an extended plasma region in the TEXTOR tokamak. This technique is made possible by collecting an extended spectrum of reflected waves with large-aperture imaging optics. Details of the imaging reflectometry concept, as well as technical details of the TEXTOR instrument will be presented. Data from proof-of-principle experiments on TEXTOR using a prototype system is presented, as well as results from a systematic off-line study of the advantages and limitations of the imaging reflectometer.
\end{abstract}

\section{INTRODUCTION AND BACKGROUND}

Microwave reflectometry has been extensively used in tokamak plasmas for the detection of turbulence, due to its relatively simple implementation and its high sensitivity to small perturbations of electron density. Despite its widespread and long-standing use, however, the interpretation of reflectometry data from fluctuations remains an outstanding issue $[1,2]$.

For the simple case of 1-D fluctuations (radial only) with a stratified plasma permittivity $\varepsilon=\varepsilon_{0}(r)+\tilde{\varepsilon}(r)$ (with fluctuating component $\tilde{\varepsilon}(r) \ll 1$ ) probed by a wave propagating in the $r$ direction, the interpretation of reflectometry is relatively simple. In this case, the fluctuating component of the signal phase is given by the approximation of geometric optics [3]

$$
\tilde{\phi}=k_{0} \int_{0}^{r_{c}} \frac{\tilde{\varepsilon}(r)}{\sqrt{\varepsilon_{0}}} d r
$$

as long as the radial fluctuation wavenumber satisfies the condition $k_{r}<k_{0} /\left(k_{0} L_{\varepsilon}\right)^{1 / 3}$, where $L_{\varepsilon}=\left(d \varepsilon_{0} / d r\right)_{r=r_{c}}^{-1}$ is the scale length of the plasma permittivity at the plasma cutoff $r=r_{c}$ and $k_{0}$ is the wavenumber of the probing beam.

By taking $\left|k_{r}\right|>1 / L_{\varepsilon}$ (since we are interested in shortscale fluctuations) and $\varepsilon_{0}(r) \approx\left(r_{c}-r\right) / L_{\varepsilon}$ (since most of the contribution to $\tilde{\phi}$ comes from a narrow region near the cutoff), one obtains the power spectrum of $\tilde{\phi}$ as a function of the power spectrum of the density fluctuations [1]

$$
\Gamma_{\phi}\left(k_{r}\right)=\pi M \frac{k_{0}^{2} L_{n}}{\left|k_{r}\right|} \Gamma_{n}\left(k_{r}\right)
$$

where $L_{n}=n /(d n / d r)_{r=r_{c}}$ is the scale length of the electron density $n, M \equiv(n d \varepsilon / d n)_{r=r_{c}}(\approx 1$ for the ordinary mode and $\approx 2$ for the extraordinary mode), $\Gamma_{\phi}\left(k_{r}\right)$ is the power spectrum of the measured $\tilde{\phi}$ (considered to be a function of $\left.r_{c}\right)$, and $\Gamma_{n}\left(k_{r}\right)$ is the power spectrum of the relative plasma density fluctuation $\tilde{n} / n$.

In the presence of 2-D turbulent fluctuations, the interpretation of reflectometry becomes considerably more complex. Unfortunately, this is precisely the case of interest for tokamak plasmas, which exhibit both radial and poloidal fluctuations. The difficulty arises from the fact that when the plasma permittivity fluctuates perpendicularly to the direction of propagation of the probing wave, the spectral components of the reflected field propagate in different directions. This can result in a complicated interference pattern on the detector plane, from which it is very difficult to extract any information about the plasma fluctuations. In essence, the measurement of the fluctuations can be limited by the fluctuations themselves.

Several papers have taken these phenomena into account by expressing an upper-bound on the measurable fluctuation levels in particular experiments. Additionally, various techniques have been employed to account for the effects of 2-D turbulence, at least for moderate fluctuation levels. In one such technique, the reflectometer signals are adjusted by numerically back-projecting the measured complex electric fields through the modeled dispersive plasma medium, providing a correction to the field distribution [4]. In the limit of strong fluctuations, however, the signal can become distorted beyond repair even by advanced numerical techniques.

The study of this problem, both on TFTR and in a series of numerical simulations, led to the development of the Microwave Imaging Reflectometry concept $[3,5]$. In this technique, large-aperture optics at the plasma edge are used to collect as much of the scattered wavefront as 
possible and optically focus an image of the cutoff layer onto an array of detectors, thus restoring the integrity of the phase measurement. A detailed overview of the technique is provided in Ref. [5], and only a basic description is provided here. The first experimental implementation of the MIR approach is underway on the TEXTOR tokamak, and is the subject of this paper.

Although much of the subject of this paper describes the application of MIR to a single point measurement, it should be noted that with the use of large optics, MIR is implicitly capable of multi-point measurements. Indeed, the application of MIR represents the first use of reflectometry measurements to simultaneously resolve the turbulent $\omega$ and $k_{\theta}$ (frequency and poloidal wavenumber) spectra using locally sampled plasma areas.

An important result from the numerical simulations in Ref. [3] which is critical to the implementation of the MIR technique is the demonstration of a "virtual cutoff" surface, located behind the actual cutoff surface, from which the reflected waves appear to have originated (to an observer at the plasma edge). The location of the virtual cutoff can be heuristically described as the intersection of the asymptotes of the ray trajectories of the probing wave before and after reflection, shown schematically in Fig. 1. If the reflected rays are collected by a large-aperture optical system with its object-plane located at the virtual cutoff, the spatial structure of the density fluctuations at the actual cutoff layer can be determined by a the detected phase at the image plane.

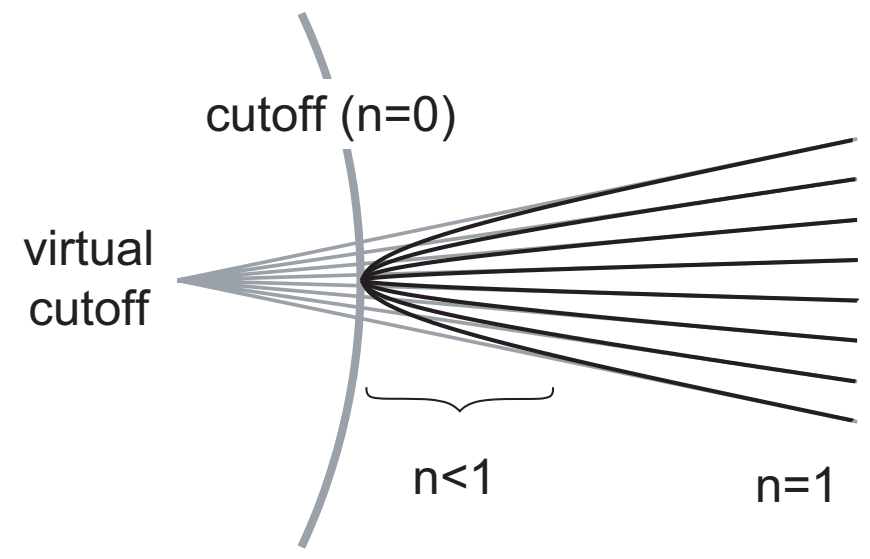

FIG. 1: Heuristic description of the virtual cutoff layer. Rays refract near the plasma cutoff layer $(n<1)$, reaching a turning point at the cutoff $(n=0)$. The ray asymptotes meet at a common location, where, to an outside observer, the radiation appears to have originated.

The distance between the actual and virtual cutoff layers was calculated to lowest order for planar geometry in Ref. [5], and is given by $\Delta r \approx \int_{r_{c}}^{r_{b}} \frac{1-\sqrt{\varepsilon(r)}}{\sqrt{\varepsilon(r)}} d r$. A practical approximation for this distance has been derived in [6] for cylindrical geometry, as a function of the radius of the cutoff surface $r_{c}$ and the scale length of the plasma permittivity $L_{\varepsilon}$ at the cutoff. In this case, $\Delta r \approx a L_{\varepsilon}{ }^{b}$ where $a=1-0.89 \exp \left(-0.43 r_{c}\right), b=1-0.66 \exp \left(-0.45 r_{c}\right)$, and all dimensions are in [m].

The remainder of this paper is organized as follows: Section II provides an overview of the issues specific to the TEXTOR instrument, including subsections which specifically address the optical design, the probing microwave beam, the frequency-selective dichroic plate, and the detector array. Section III contains results from a series of off-line laboratory tests which assess the performance of the TEXTOR MIR instrument. While routine data will not be available until the resumption of TEXTOR operation in October 2002 (following an 18-month maintenance/upgrade period), a prototype of the MIR system was installed on TEXTOR prior to its extended shutdown. An example of data from that series of experiments is presented in section IV, followed by short summary and overview of future plans in section V.

\section{APPLICATION TO TEXTOR}

\section{A. Overview}

For the TEXTOR instrument, shown schematically in Figure 2, the primary focusing optical set is composed of two large cylindrical mirrors, arranged to tailor the illumination beam wavefront to match the toroidal cutoff surface. The probing and reflected beams enter and exit the vacuum chamber through the same window, and share the primary mirrors. The reflected beam is separated from the probing beam with a beamsplitter, and is focused onto a 16-element linear array of detectors. The MIR system has been combined with an Electron Cyclotron Emission Imaging diagnostic [7], which shares the $42 \mathrm{~cm} \times 20 \mathrm{~cm}$ vacuum window and large front-end optics, enabling simultaneous measurement of $\tilde{n}_{e}$ and $\tilde{T}_{e}$ fluctuations in the same plasma volume. A dichroic plate is used to separate the higher frequency $(>110 \mathrm{GHz})$ ECEI signal from the lower frequency $(<90 \mathrm{GHz}) \mathrm{MIR}$ signal, and each subsystem uses a dedicated detector array.

TEXTOR will employ a wide range of operating scenarios during the upcoming campaign, including Dynamic Ergodic Divertor operation at $1.9 \mathrm{~T}$ and higher, several scenarios at 2.25-2.35 T including ECH studies using a $110 \mathrm{GHz}$ gyrotron, and additional ECH studies at 2.5-2.6 T using a $140 \mathrm{GHz}$ gyrotron.

The MIR instrument, installed initially at a fixedfrequency of $88 \mathrm{Ghz}$, covers a $\lesssim 15 \mathrm{~cm}$ poloidal region of the cutoff surface with a spatial resolution of $\sim 1 \mathrm{~cm}$, leading to a theoretical $k_{\theta}$ resolution of $0.4 \mathrm{~cm}^{-1} \leq k_{\theta} \leq$ $3 \mathrm{~cm}^{-1}$. It is important to specify the distinction between making measurements in the presence of poloidal fluctuations and making measurements of the poloidal fluctuations. While the majority of the effort behind the development of the MIR technique has been concerned with the validity of measurements in the presence of poloidal fluctuations, in fact the MIR project repre- 


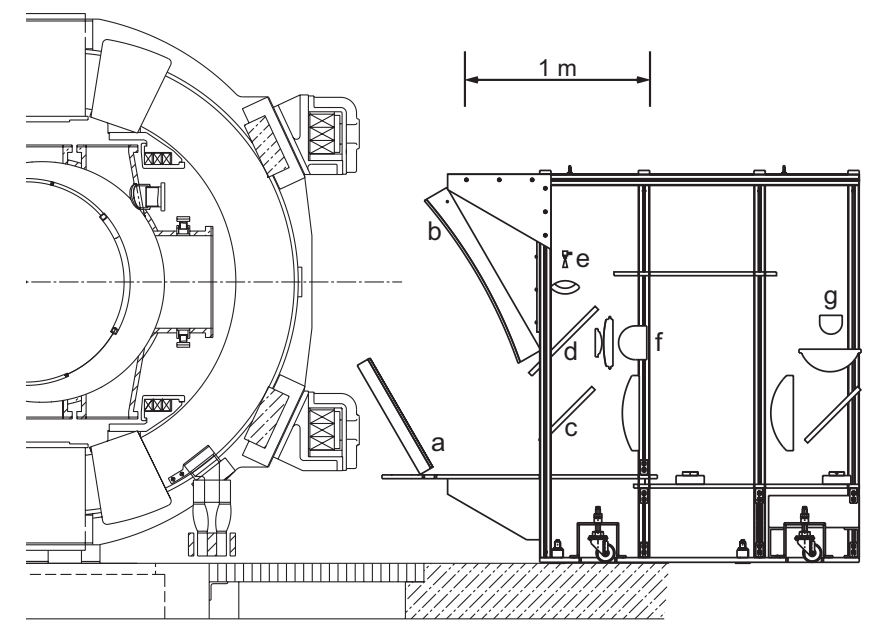

FIG. 2: TEXTOR poloidal cross-section with the MIR/ECEI combined system. Visible in the diagram are the (a) toroidal and (b) poloidal focusing mirrors, (c) dichroic plate, (d) beamsplitter, (e) probing beam source, (f) MIR detector array, and (g) ECEI detector array, as well as several focusing lenses.

sents the first use of simultaneous, localized reflectometry measurements to produce a time-resolved mapping of $k_{\theta}$. With this in mind, the stated $k_{\theta}$ resolution of the TEXTOR MIR system refers to that which can actually be resolved by the instrument. Measurements can be made in the presence of arbitrarily low $k_{\theta}$, down to $0 \mathrm{~cm}^{-1}$.

In future experiments, simultaneous radial and poloidal correlation measurements will be made available with the addition of a second, scanning-frequency probing beam and an upgraded detection system.

\section{B. Optical Design}

The large toroidal and poloidal mirrors, while primarily used for the reflection and focusing of microwaves, nonetheless were constructed to be optical quality to facilitate system alignment using lasers. The mirror surfaces are made from a polystyrene-backed aluminum film bonded to a machined substrate. The substrate is made from non-metallic composite to avoid eddy-currents from transient magnetic fields. Focusing mirrors were chosen rather than lenses for the front-end elements to avoid internal reflections from the lens surfaces, which could potentially interfere with the plasma signal.

Although the primary optics are shared between the probing and the reflected beam, the optical elements are not performing the same function during illumination and detection. For the probing beam, the role is to tailor the wavefront to the shape of the cutoff surface, thus making the wavefront impinge normally the cutoff surface. This increases the robustness of the optical system to changes in the size of the cutoff surface and minimizes the deleterious effect of plasma refraction on the spectrum of probing wave numbers [5]. To perform this function, the optics must have a focal point in the toroidal direction located at the center of the torus, and in the poloidal direction at the center of curvature of the cutoff surface. By using the curvature-matching technique, cutoff surfaces over a wide range of densities can be illuminated with minimal change to the optics.

The function of the optical system for the reflected waves is to form an image of the virtual cutoff onto an array of detectors. The design must take into account the effect of apertures on the minimum achievable beam size. To maximize the power density arriving at the detector, it is desireable to demagnify the image as much as possible, consistent with the detector element size and the predicted maximum resolution in the plasma. In addition to the focusing mirrors, several lenses were used to improve the image quality and match the image to the detector characteristics (see Fig. 2). All lenses are made from high-density polyethylene (HDPE), and are placed behind the beam-splitter, out of the path of the probing beam. Several of the HDPE lenses are designed with different curvatures in the E-plane and H-plane, to compensate for the different focal points of the two primary mirrors.

The primary constraint on the system resolution is imposed by the vacuum-window aperture, and all other optical elements are sized to avoid further vignetting of the beam. Using the fact that the detectors impose a nearly Gaussian profile on the collected radiation (see Figure 6), the best instrument resolution is determined by the Gaussian spot-size at the cutoff, given by $2 r_{\text {spot }}=2 \lambda_{0} d / \pi h$, where $r_{\text {spot }}$ is the Gaussian beam waist at the object plane, $\lambda_{0}$ is the wavelength of the probing beam, $d$ is the distance from the aperture to the beam waist, and $h$ is the $21 \mathrm{~cm}$ half-height of the aperture. Clearly it is possible for the instrument resolution to be different in the toroidal and poloidal directions, though it has been assumed that the E-plane (poloidal) resolution is of primary concern. For the central channels, the poloidal angular acceptance of reflected radiation fan is $\pm 12^{\circ}$ about the specularly reflected ray.

Off-axis channels are degraded from the central channel by vignetting of the reflected radiation, which effectively reduces the available aperture. The degree of reduction increases from the center to the edge, reaching $50 \%$ at the outermost channels. This effect not only degrades the poloidal resolution, but also reduces the returned power, depending on the degree of spreading of the ray fan. Assuming a fan which spreads to fill the entire aperture accepted by the antennas, this effect increases linearly from the center to the edge, also reaching $50 \%$ at the outermost channels. For narrower ray-fans, this effect will be reduced.

Figure 3a shows the Gaussian spot-radius, plotted vs. poloidal position in the plasma, calculated using the set of Gaussian beams which begin at each plasma position and propagate through the entire optical system, including the receiver antennas. Figure $3 \mathrm{~b}$ shows the relative 
power profile of the illumination and reflected beams, based on the vignetting effects described above.

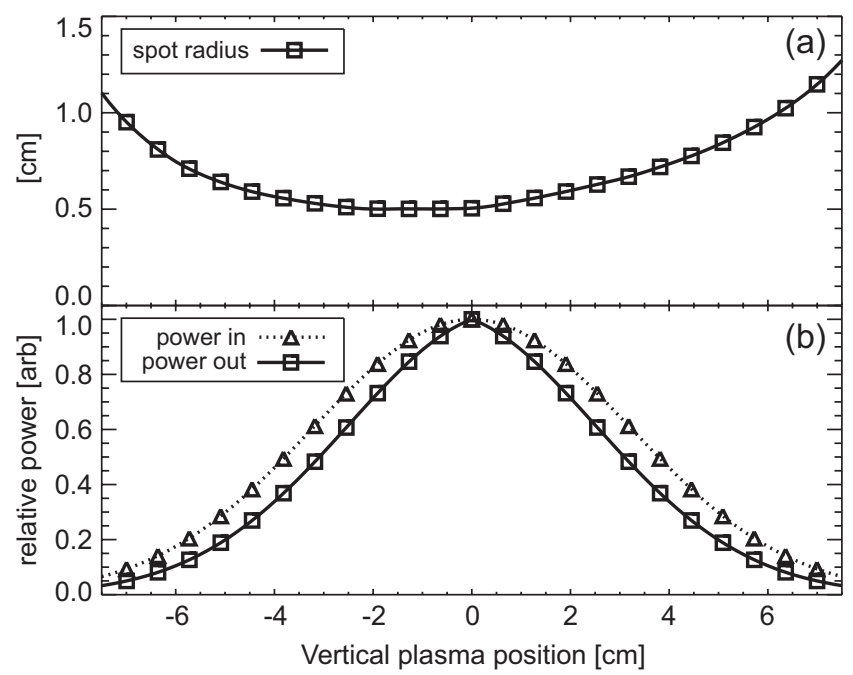

FIG. 3: Minimum Gaussian spot radius (a) and relative forward/return probing beam power (b), calculated by considering the effects of vignetting by the vacuum window, focusing elements, and detector acceptance patterns. Discrete markers (squares and triangles) represent the location of the detector array elements, mapped back to the plasma cutoff surface.

In both Figs. 3a and 3b, the discrete markers (squares and triangles) represent the location of the detector array elements, mapped back to the plasma cutoff surface. Although there are 23 channels shown, the interchannel spacing can be compared to the resolution from Fig. 3a, and it is clear that the plasma is slightly oversampled, increasing towards the edge. Also observable in Fig. 3a is a top/bottom asymmetry to the spot-size distribution, which results from the vertically-asymmetric optical configuration (see Fig. 2).

\section{Probing Beam}

In order to minimize the phase variation introduced by the probing beam itself, it is desirable to launch as clean a Gaussian beam as possible, with minimized sidelobes, and maintain the clean beam through the focusing elements. Figures 4a-c show the beam pattern from the specialized launching horn, demonstrating the high degree of purity of the Gaussian profile. Figure 4a is the 2-D pattern measured in front of the horn, and Figures $4 \mathrm{~b}$ and $4 \mathrm{c}$ are 1-D "slices" in the $\mathrm{y}$ and $\mathrm{x}$ direction, respectively. Here, squares represent single-point measurements, and solid lines represent the best-fit Gaussian profile. In Figure 4d, the squares represent measured beam widths at various distances along an optical path through a series of focusing optics, and the solid line represents the theoretical prediction of the Gaussian width propagating through the system.
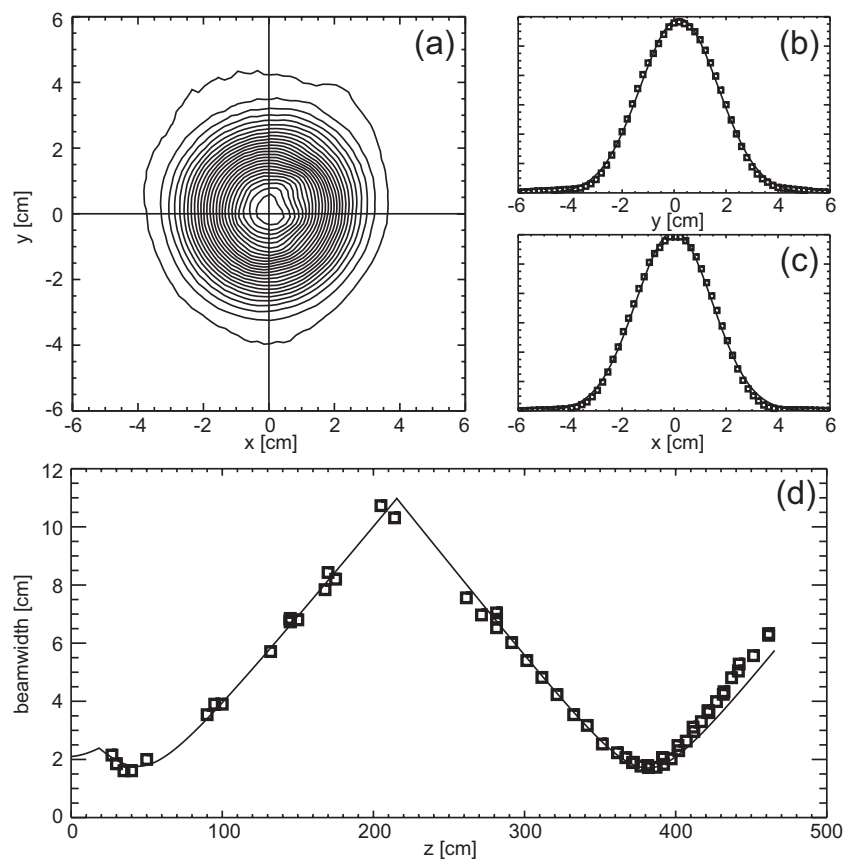

FIG. 4: Measured 2-D profile of the launched Gaussian probing beam (a), shown with 1-D "slices" in the y and x direction ( $b$ and c, respectively), and the measured beam waist through the primary focusing mirror (d). In (d), squares represent measured beam widths at points along the optical path, and the solid line represents the theoretical prediction.

\section{Dichroic Plate}

In order to enable simultaneous measurement of $\tilde{n}_{e}$ and $\tilde{T}_{e}$ fluctuations using a common mirror set, it is necessary to split the MIR signal from the ECEI signal, and send each to a dedicated detector array. For this purpose a dichroic plate was designed and constructed to reflect the MIR signal at $88 \mathrm{GHz}$ while transmitting ECEI radiation over a range of 114-140 GHz. The design and fabrication of dichroic plates for use at normal incidence are well established and fairly straigtforward, requiring either square grids or arrays of round holes [8]. The MIR/ECEI system geometry necessitates a high angle of incidence, however, which requires a somewhat more complicated plate design comprised of an array of oblong holes arranged on a rectangular grid [9].

Figure 5a is a schematic of a small section of the hole grid, comprised of $1.654 \times 0.717 \mathrm{~mm}$ oblong holes, separated by $0.22 \mathrm{~mm}$ in both dimensions, cut from a plate 2.0 $\mathrm{mm}$ thick $[9,10]$. Figure $5 \mathrm{~b}$ shows the calculated transmission and reflection characteristics of the plate for $45^{\circ}$ incidence, demonstrating both the nearly complete reflection at $88 \mathrm{GHz}$ and the nearly unity transmission band from 114-140 GHz. At the time of this publication, the plate is still in the testing stage, and no performance data is available. Theoretically, the transmission and reflection characteristics should be quite robust to incidence at angles up to $\pm 15^{\circ}$ about the nominal design incidence 
angle of $45^{\circ}$. Indeed, the characteristics of plates designed using this technique have been demonstrated to match the expected performance very closely [9].
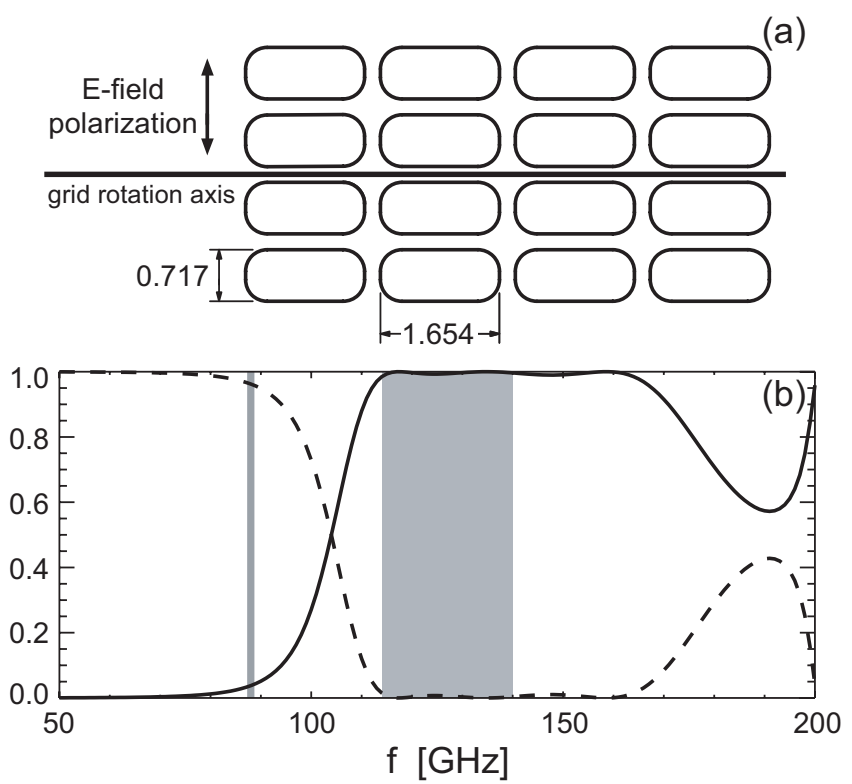

FIG. 5: (a) Schematic drawing of a small section of the dichroic plate hole grid, designed to reflect $88 \mathrm{GHz}$ and transmit $114-140 \mathrm{GHz}$ at $45^{\circ}$ incidence. (b) Calculated transmission (solid) and reflection (dashed) curves.

\section{E. Detector Array}

The viability of imaging reflectometry is dependent on the availability of sensitive detectors. In this regard there has been steady progress in the design of multichannel arrays that can be inexpensively manufactured on printed circuit boards and can therefore scale-up to large multichannel arrays $[11,12]$.

The MIR system uses a hybrid-type detector/mixer array, comprised of printed antennas coupled with beamlead diodes, which are silver epoxied onto the feeds of each array element. Frequency mixing is achieved by illuminating the front face of the array with the signal beam while the back face is illuminated by the local oscillator.

Past microwave imaging systems have used slot-bowtie antennas, though the present MIR system uses dualdipole antennas, which exhibit similar bandwidth to the slot-bowties $(\sim 15 \%)$, but have much cleaner and more symmetric antenna patterns, as well as improved sensitivity. Figure 6a shows a mask layout of the MIR dualdipole detector array, with 16 channels shown and the Eplane oriented horizontally. The center-to-center element spacing is $2.29 \mathrm{~mm}$ in the E-plane and $1.40 \mathrm{~mm}$ in the $\mathrm{H}$-plane. Figure $6 \mathrm{~b}$ is a zoom photograph of a single detector (E-plane oriented vertically), showing the details of the antenna pattern and the epoxied diode bridging the antenna halves. Figures $6 \mathrm{c}$ and $6 \mathrm{~d}$ show the E-plane and H-plane antenna patterns, respectively, demonstrating the clean and symmetric Gaussian response.
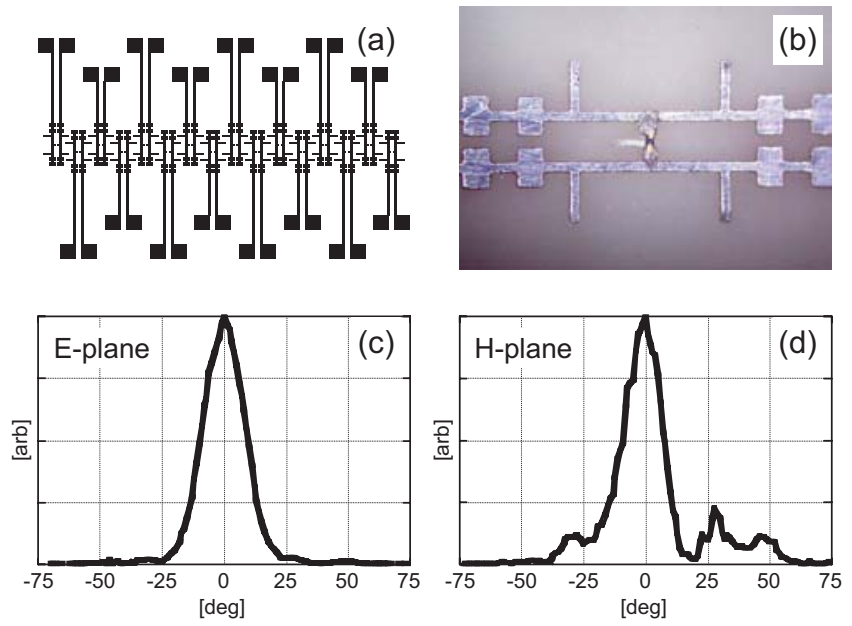

FIG. 6: Detector array layout (a), zoom photograph of single array element (b), and antenna sensitivity curves vs. angle in the E-plane (c) and H-plane (d), recorded at $89 \mathrm{GHz}$. The center-to-center element spacing is $2.29 \mathrm{~mm}$ in the E-plane and $1.40 \mathrm{~mm}$ in the H-plane.

\section{CHARACTERIZATION OF OPTICAL PERFORMANCE}

Simultaneously with the installation of the TEXTOR instrument, we have performed an off-line laboratory study of the characteristics of the MIR instrument, using corrugated reflecting targets of known shape to simulate the fluctuating plasma reflection layer. This approach was chosen to augment the plasma measurements prior to complete integration due to the unavoidable complexity of any new plasma data; implementation of any new instrument is made much more reliable by first making reference measurements. This study also included a performance characterization of a conventional reflectometer arrangement, consisting of a Gaussian launching horn and a simple detector horn with no imaging optics, also arranged to measure the reflected signals from the corrugated targets. Details of this calibration process are presented in [6], with an overview presented here. This type of arrangement has been used in the past to simulate doppler-shift measurements of poloidal rotation with reflectometry [13], and even to investigate the effects of 2-D fluctuations on reflectometry measurements [14], though in the latter case only the effects on total collected power using a 1-D configuration were considered.

The target reflectors were $60 \mathrm{~cm}$ in diameter and 20 cm wide, with a single, precisely imposed corrugation wavelength $\lambda_{\text {corr }} \equiv 2 \pi / k_{\theta}$ and corrugation height $h_{c o r r}$. Measurements were taken with each of the reflectometry systems for a series of targets covering a range of $k_{\theta}$ and $h_{c o r r}$, and for geometries covering a range of dis- 
tances from the instrument to the target surface. This distance $(d)$ is defined as the distance between the target surface to the first mirror in the case of the MIR system, and as the distance between the target surface and the launch/receive horns in the case of the 1-D system. The focal distance of the MIR system $d_{0} \equiv d$ (image focus) is $235 \mathrm{~cm}$. To form a reference measurement to which the reflectometer measurements could be compared, each target surface was independently measured using Leica "Laser Tracker" [15], a visible-laser interferometer with $10 \mu \mathrm{m}$ precision.

A result from this off-line study is shown in Fig. 7, in which the measurements from both the 1-D and MIR systems are compared to the reference measurement. The target in this case had corrugations of $k_{\theta}=1.25 \mathrm{~cm}^{-1}$ and depth $\approx 1.7 \mathrm{~mm}=\lambda_{0} / 2$, where $\lambda_{0}$ is the wavelength of the probing microwave beam, leading to a nominal phase fluctuation of $\Delta \phi \approx 2 \pi$. For TEXTOR parameters, this corresponds to $\tilde{n}_{e} / n_{e} \approx 1 \%$. In the Figure, the gray curve represents the reference measurement of the corrugation shape scaled by $4 \pi / \lambda_{0}$, corresponding to the ideal phase shift induced on the reflected beam. Each curve is plotted against the rotation angle of the target wheel. Figs. $7 \mathrm{a}$ and $7 \mathrm{~b}$ correspond to measurements taken with the 1-D system at distances of $10 \mathrm{~cm}$ and 30 $\mathrm{cm}$, respectively. Fig. 7c corresponds to measurements taken with the MIR system located at $d_{0}$, at the focal distance of $235 \mathrm{~cm}$.

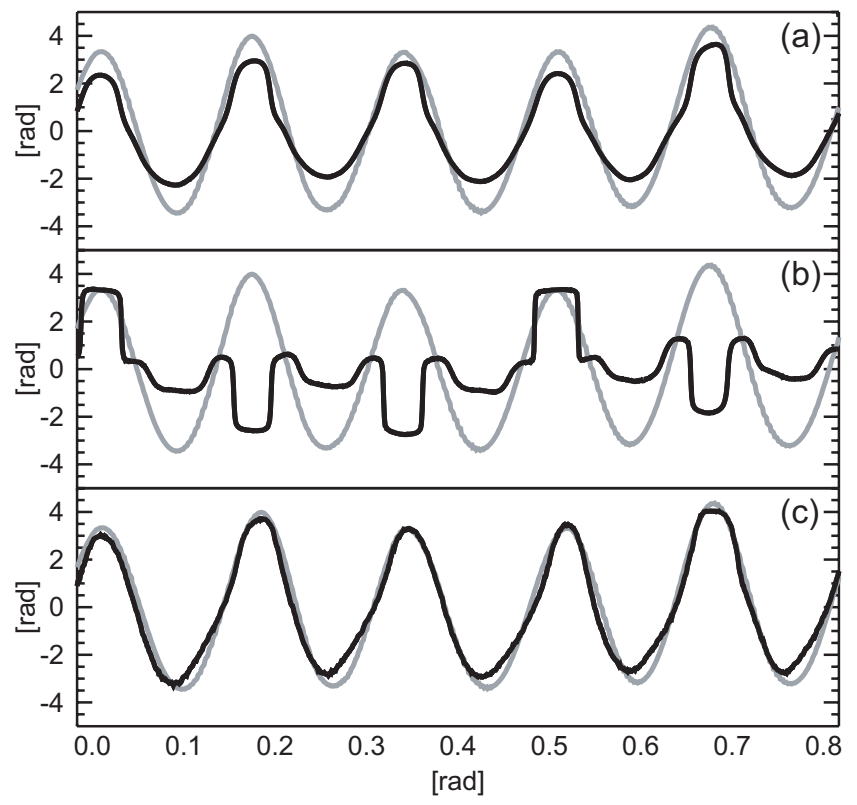

FIG. 7: Waveforms from the 1-D system (a,b) and MIR system (c), from measurements of a target reflector having corrugations of $k_{\theta}=1.25 \mathrm{~cm}^{-1}$ and depth $\approx 1.7 \mathrm{~mm}$, leading to $\Delta \phi \approx 2 \pi$. The solid curves are the reflectometer measurements, and the gray curve is the reference measurement. Plots (a) and (b) are measurements taken with the 1-D system at distances of $10 \mathrm{~cm}$ and $30 \mathrm{~cm}$, respectively, and (c) is a measurement taken with the MIR system located at the focal distance of $235 \mathrm{~cm}$.
Clearly from Fig. 7a, the 1-D configuration produces a very close match to the reference curve, although it appears that some minor level of interference has reduced the measured level of phase modulation compared to the actual surface. It is obvious that the majority of the spectral power is contained in the fundamental $k_{\theta}$ of the target wheel. The 1-D measurement at $30 \mathrm{~cm}$ (Fig. 7b), however, is dramatically distorted, no longer representing the target surface. Clearly a significant fraction of the spectral power in this plot is contained in higher harmonics of $k_{\theta}$, and the wheel shape (which represents the fluctuations at the plasma cutoff), can not be inferred from the reflectometer data in this case.

The MIR measurement (Fig. 7c) represents the cleanest measurement of the target surface, despite being physically the furthest removed. Even the small irregularities in the reference curve (due to construction irregularities in the target wheel) are reproduced by the MIR measurement.

In order to quantify the degree to which the reflectometer measurements accurately reproduced the reference surface, the cross-correlation coefficient $\rho_{X Y}$ was calculated between the power spectra of the reflectometer and reference curves for measurements over a wide range of $d$, the distance between the instrument and the target surface [16]. This data, plotted for both the 1-d and MIR systems, is shown in Fig. 8a.

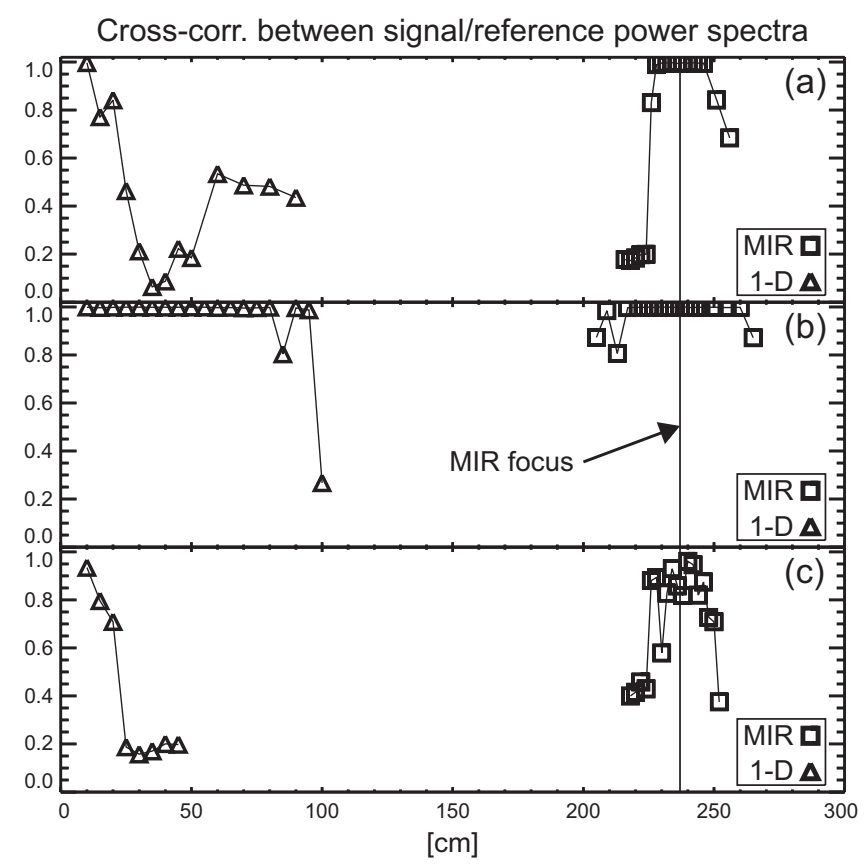

FIG. 8: Cross-correlation between the reflectometer and reference power spectra, plotted for both the 1-D and MIR configurations, for 3 target reflectors with the following corrugation values: (a) $k_{\theta}=1.25 \mathrm{~cm}^{-1}$ and $\Delta \phi \approx 2 \pi$; (b) $k_{\theta}=1.25 \mathrm{~cm}^{-1}$ and $\Delta \phi \approx \pi$; (c) $k_{\theta}=2.5 \mathrm{~cm}^{-1}$ and $\Delta \phi \approx 0.7 \pi$.

In the Figure, the triangles represent the 1-D measurements, and the squares represent the MIR measurements. 
Figure 8 a represents data taken with the target from Fig. 7 , having $k_{\theta}=1.25 \mathrm{~cm}^{-1}$ and $\Delta \phi \approx 2 \pi$. Figure 8b represents a second target, also with $k_{\theta}=1.25 \mathrm{~cm}^{-1}$ but with $\sim 50 \%$ lower corrugation depth $(\Delta \phi \approx \pi)$. Figure 8c represents a target with $k_{\theta}=2.5 \mathrm{~cm}^{-1}$, higher than the preceding two targets by a factor of two, and $\Delta \phi \approx 0.7 \pi$, somewhat lower than either of the preceding two targets.

In Figure 8a, for the 1-d case, the correlation is nearly unity for $\mathrm{d}=10 \mathrm{~cm}$, and falls quickly as the distance is increased to $30 \mathrm{~cm}$ or more. As was seen in Fig. 7, measurements at or beyond $30 \mathrm{~cm}$ no longer represent the actual surface, as represented by the $\leq 0.5$ cross-correlation figure. The MIR values are similarly near-unity in the vicinity of the MIR focus, falling off at $d \approx \pm 10 \mathrm{~cm}$ with respect to the focal plane location. This $20 \mathrm{~cm}$ range represents the distance over which multi-radial (multifrequency) data could be collected simultaneously with a fixed set of imaging optics. This plot serves to illustrate the fundamental advantage of the MIR technique, which is that the "proximity focusing" of the 1-D system for data taken immediately next to the reflecting surface is transferred to a remote focal plane, physically accessible to a detection system.

In Fig. 8b, the case with reduced corrugation level, the response of both the 1-D and MIR systems is improved over the previous case. Both reflectometry configurations exhibit near-unity cross-correlation between the measured and reference power spectra, indicating that for these conditions, the measurement of fluctuations has essentially been reduced to the 1-D problem, and is free from interference effects. Indeed, for the case of Fig. 8b the need for imaging is eliminated.

In Fig. $8 \mathrm{c}$, the case with the highest $k_{\theta}$, the signal quality is degraded with comparison to the previous targets. Additionally, the "depth of field" of acceptable levels of cross-correlation is slightly reduced for both systems. As in the previous cases, the similarity between the focal depth of the 1-D system and the MIR system is apparent. The reflectometer signal quality and the resulting need for MIR is critically dependent on the target $k[3,6,17]$.

In order to better understand the details of the target reflector measurements with the 1-D and imaging systems, the reflected field was analytically calculated using a solution to Maxwell's equations in cylindrical geometry. It was found that the calculated waveforms were a very accurate match to the data, even in the cases where the amplitude waveforms were quite complicated and the phase waveforms exhibited a high level of distortion.

Even in cases where the interference-dominated waveform appears to be quasi-periodic, the addition of even a single additional mode (which causes a relatively small change in the shape of the reflector) results in a dramatic change in the detected interference pattern, which quickly becomes chaotic [6]. Because of this, in cases where interference plays a significant role in the detected signal, modeling the effect of interference by looking at a single $k_{\theta}$ mode can be overly optimistic. At the same time, any direct quantitative comparison of absolute fluc- tuation levels between the target-reflector tests and realistic plasma conditions is necessarily imprecise, since in the former case all of the spectral energy is in a single mode, and in the latter case the energy is spread over the $k_{\theta}$ spectrum.

The implication from the target wheel tests is that for low $k_{\theta}$ and $h_{c o r r}$, imaging is unnecessary, and 1-D reflectometry can be expected to produce valid fluctuation measurements. If either (or both) of these quantities is increased beyond some threshold, however, the degradation of the signal quality due to wave interference becomes an important consideration. While the exact threshold level depends on the details of the profile and the experimental arrangement, the experimental observations on TFTR as well as the implications from the corrugated target tests indicate that this level lies precisely in the region of interest for core measurements of tokamak plasmas. As a rough guide, one can expect interference to play a significant role in the reflected field pattern if measurements are taken beyond the diffraction distance, defined as $D_{\text {diff }} \approx \frac{2 k_{0}}{\left\langle(\Delta \phi)^{2}\right\rangle\left\langle k_{\theta}\right\rangle^{2}}$, where $\sqrt{\left\langle(\Delta \phi)^{2}\right\rangle}$, is the average phase deviation of the measurement [17].

It is important to reiterate that the target-reflector tests represent only a simplified simulation of the plasma turbulence, chosen to illustrate the effect of 2-D fluctuations in the simplest possible manner. The inclusion of a more realistic spectrum containing many modes and refractive effects in the plasma can only further complicate the data and its interpretation. That said, the targetreflector tests constitute a minimum first test of the viability of the new instrument, and serve to illuminate the limitations of both imaging and non-imaging reflectometry at a most basic level.

\section{RESULTS FROM TEXTOR}

Preceding the extended TEXTOR maintenance period, a prototype MIR system was installed, taking advantage of a refractive optical set previously used for an ECEI instrument. The proof-of-principle results are presented in Reference [18], with only a single highlight presented here.

For this experiment, the probing frequency and optics were held fixed, while the plasma density was slowly ramped over the course of the shot. In this way the cutoff layer was brought from a position outside the focal range of the MIR instrument through the focal region and beyond on the opposite side. Figures $9 \mathrm{a}$ and $9 \mathrm{~b}$ show two example I/Q plots from a single channel recorded over 3 msec time windows as the central plasma density was ramped from $2.8 \times 10^{19} \mathrm{~m}^{-3}$ at $\mathrm{t}=1.2 \mathrm{~s}$ (Fig. 9a), to $4.4 \times 10^{19} \mathrm{~m}^{-3}$ at $\mathrm{t}=1.9 \mathrm{~s}$ (Fig. 9b). During the density ramp, the cutoff position moved from 1.93 to $1.99 \mathrm{~m}$ for the plots shown. At $\mathrm{t}=2.4 \mathrm{~s}$ (not shown), the cutoff position had moved out to $2.06 \mathrm{~m}$, producing an I/Q signal indistinguishable from Fig. 9a.

The striking difference between plots $9 \mathrm{a}$ and $9 \mathrm{~b}$ is in 

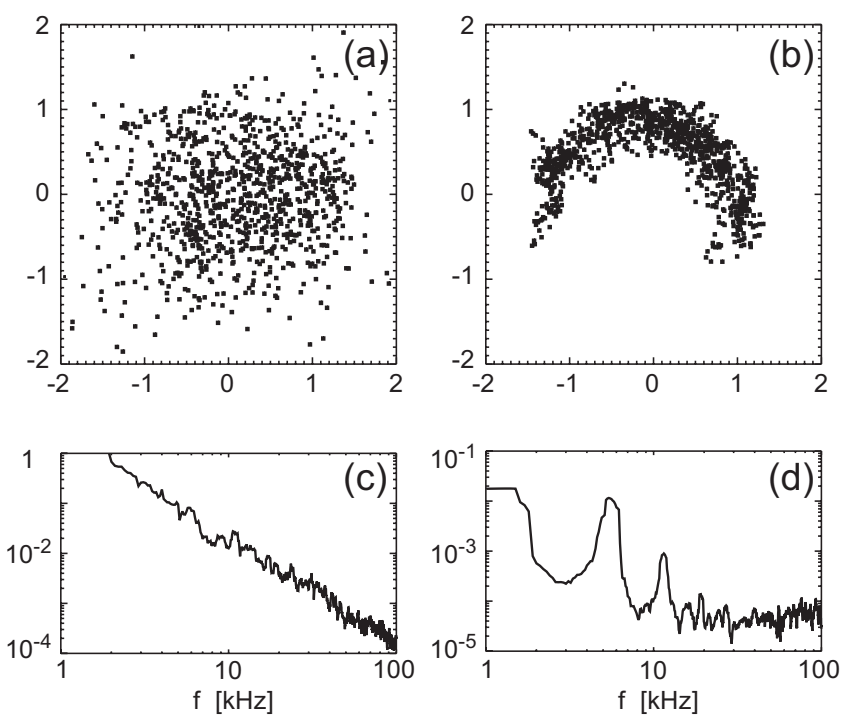

FIG. 9: I/Q plots from the prototype TEXTOR MIR instrument, for (a) out-of-focus and (b) in-focus conditions. Also, the corresponding power spectra of the phase waveforms $(c, d)$.

the level of amplitude fluctuations, which is much larger in the former case. These data were collected in ohmic plasmas where, apart from a small density rise, all plasma parameters were stationary. Thus, consistent with the off-line tests of the MIR instrument, it appears that reduced amplitude fluctuations in plot $9 \mathrm{~b}$ are due to the in-focus condition of the cutoff. This is corroborated by the agreement between the calculated position of the vir- tual cutoff in plot $9 \mathrm{~b}$ with the position of the instrument focal plane.

The power spectra for the two cases, shown in Figures 9c and 9d, are also quite different. In Fig. 9d (in focus), the spectrum is dominated by large coherent MHD fluctuations, while for Fig. 9c (out of focus), is a featureless $1 / f^{2}$ spectrum, exhibiting not even the slightest indication of the dominant MHD fluctuations visible in Fig. 9d.

\section{DISCUSSION}

Although we have just begun to exploit the potential of the imaging reflectometer, the combination of numerical simulation, off-line system testing, and preliminary plasma data from TEXTOR give us confidence in the capability of this technique to deliver fluctuation data at a level unattainable up to now. Future upgrade plans to the TEXTOR MIR system include the extension to multiple frequencies, which will enable simultaneous radially and poloidally resolved data. Additionally, MIR instruments will be installed on other tokamak devices as space becomes available.

\section{Acknowledgments}

The authors would like to thank the TEXTOR team for their continuing support of this project. This work was supported by U.S. DOE contracts DE-AC0276CH03073, DE-FG02-99ER54523 and the U.S. DOE Fusion Energy Postdoctoral Fellowship.
[1] E. Mazzucato and R. Nazikian, Phys. Rev. Lett. 71, 1840 (1993).

[2] R. Nazikian and E. Mazzucato, Rev. Sci. Instrum. 66, 392 (1995).

[3] E. Mazzucato, Rev. Sci. Instrum. 69, 1691 (1998).

[4] R. Nazikian, J. Mod. Opt. 44, 1037 (1997).

[5] E. Mazzucato, Nucl. Fus. 41, 203 (2001).

[6] T. Munsat, E. Mazzucato, H. Park et al., Plasma Phys. and Contr. Fus. 999, 9999 (2003), to be published.

[7] B. Deng, C. Domier, N. C. Luhmann, Jr., D. Brower, A. Donné, T. Oyevaar, and M. van de Pol, Phys. Plasmas 8, 2163 (2001).

[8] C. Chen, IEEE Trans. Micr. Theory Tech. MTT-21, 1 (1973).

[9] P. Siegel, R. Dengler, and J. Chen, IEEE Micr. Guid. Wav. Lett. 1, 8 (1991).

[10] P. Siegel, 2001, private communication.

[11] R. Hsia, W. Geck, S. Cheng, W. Zhang, C. Domier, and N. C. Luhmann, Jr., Rev. Sci. Instrum. 66, 834 (1995).
[12] P. Hsu, B. Deng, J. Wang, C. Domier, and N. C. Luhmann, Jr., Rev. Sci. Instrum. 66, 834 (1995).

[13] O. Pavlichenko, A. Skibenko, I. Fomin, I. Pinos, V. Ocheretenko, and V. Berezhniy, in Proceedings of the $5^{\text {th }}$ International Workshop on Reflectometry, edited by K. Kawahata (National Institute for Fusion Science, Oroshicho, Toki 509-5292, Japan, 2001), No. NIFS-PROC-49, p. 85.

[14] G. Conway, Rev. Sci. Instrum. 64, 2782 (1993).

[15] Leica Geosystems AG, Mönchmattweg 5 CH-5035 Unterentfelden, Switzerland www.leica-geosystems.com.

[16] J. S. Bendat and A. G. Piersol, Random Data : analysis and measurement procedures, 3rd ed. (John Wiley \& Sons, New York, 2000).

[17] E. Mazzucato and R. Nazikian, Rev. Sci. Instrum. 66, 1237 (1995).

[18] E. Mazzucato, T. Munsat, H. Park et al., Phys. Plasmas 9, 1955 (2002). 


\section{External Distribution}

Plasma Research Laboratory, Australian National University, Australia

Professor I.R. J ones, Flinders University, Australia

Professor J oão Canalle, Instituto de Fisica DEQ/IF - UERJ , Brazil

Mr. Gerson O. Ludwig, Instituto Nacional de Pesquisas, Brazil

Dr. P.H. Sakanaka, Instituto Fisica, Brazil

The Librarian, Culham Laboratory, England

Library, R61, Rutherford Appleton Laboratory, England

Mrs. S.A. Hutchinson, JET Library, England

Professor M.N. Bussac, Ecole Polytechnique, France

Librarian, Max-Planck-Institut für Plasmaphysik, Germany

J olan Moldvai, Reports Library, MTA KFKI-ATKI, Hungary

Dr. P. Kaw, Institute for Plasma Research, India

Ms. P.J . Pathak, Librarian, Insitute for Plasma Research, India

Ms. Clelia De Palo, Associazione EURATOM-ENEA, I taly

Dr. G. Grosso, Instituto di Fisica del Plasma, Italy

Librarian, Naka Fusion Research Establishment, J AERI, J apan

Library, Plasma Physics Laboratory, Kyoto University, J apan

Research Information Center, National Institute for Fusion Science, J apan

Dr. O. Mitarai, Kyushu Tokai University, J apan

Library, Academia Sinica, Institute of Plasma Physics, People's Republic of China

Shih-Tung Tsai, Institute of Physics, Chinese Academy of Sciences, People's Republic of China

Dr. S. Mirnov, TRINITI, Troitsk, Russian Federation, Russia

Dr. V.S. Strelkov, Kurchatov Institute, Russian Federation, Russia

Professor Peter Lukac, Katedra Fyziky Plazmy MFF UK, Mlynska dolina F-2, Komenskeho Univerzita, SK-842 15 Bratislava, Slovakia

Dr. G.S. Lee, Korea Basic Science Institute, South Korea

Mr. Dennis Bruggink, Fusion Library, University of Wisconsin, USA

Institute for Plasma Research, University of Maryland, USA

Librarian, Fusion Energy Division, Oak Ridge National Laboratory, USA

Librarian, Institute of Fusion Studies, University of Texas, USA

Librarian, Magnetic Fusion Program, Lawrence Livermore National Laboratory, USA

Library, General Atomics, USA

Plasma Physics Group, Fusion Energy Research Program, University of California at San Diego, USA

Plasma Physics Library, Columbia University, USA

Alkesh Punjabi, Center for Fusion Research and Training, Hampton University, USA

Dr. W.M. Stacey, Fusion Research Center, Georgia Institute of Technology, USA

Dr. J ohn Willis, U.S. Department of Energy, Office of Fusion Energy Sciences, USA

Mr. Paul H. Wright, Indianapolis, Indiana, USA 
The Princeton Plasma Physics Laboratory is operated by Princeton University under contract with the U.S. Department of Energy.

\author{
Information Services \\ Princeton Plasma Physics Laboratory \\ P.O. Box 451 \\ Princeton, NJ 08543
}

Phone: 609-243-2750

Fax: 609-243-2751

e-mail: pppl_info@pppl.gov

Internet Address: http://www.pppl.gov 